\title{
HERRAMIENTAS ACTUALES PARA EL DIAGNÓSTICO, MANEJO Y CONTROL DE LA CARIES DENTAL. PARTE II. UNA REVISIÓN DE LA LITERATURA
}

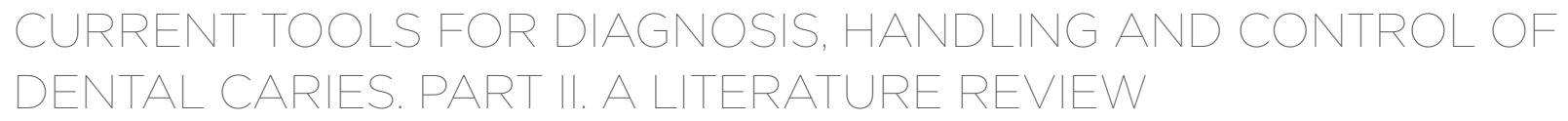

Jackeline Jajaira Jara-Porroa ${ }^{*}$ (i) jacky.jara.p@gmail.com

Gabriela Silvia De la Cruz-Sedano' gadelacruz17@gmail.com

Abigail Katherine Ventura-Flores' abigail.katherine@hotmail.com

Guido Alberto Perona-Miguel de Priego' guidoperona54@gmail.com

Artículo recibido: 12/11/2019

Arbitrado por pares

Artículo aceptado: 26/03/2020

* Autor corresponsal:

Jackeline Jajaira Jara-Porroa

jacky.jara.p@gmail.com

\section{RESUMEN}

El Sistema Internacional de Clasificación y Manejo de la Caries (ICCMS) es un conjunto de protocolos clínicos basados en tener un mejor enfoque en el diagnóstico, tratamiento y prevención de la caries dental. Consiste en modificar los factores de riesgo y tratar los dientes con lesiones de caries activas para preservar la estructura del diente y restaurarlo solo cuando sea necesario.

El objetivo de este estudio es presentar las herramientas de diagnóstico actuales para mejorar el proceso de toma de decisiones al seleccionar un plan de tratamiento y permitirnos una atención más individualizada en cada paciente.

Palabras clave: caries dental, diagnóstico, ICDAS, ICCMS

\section{ABSTRACT}

The International System of Classification and Management of Caries (ICCMS) is a comprehensive set of clinical protocols aimed at providing a better approach to the diagnosis, treatment and prevention of dental caries.

Modification of risk factors, treatment of teeth with active caries lesions and the preservation of the structure of dental enamel in order to restore tooth structure only when necessary are ideal approaches that odontopediatricians should incorporate into daily practice.

The objective of this study was to present the current diagnostic tools to improve the decisionmaking process when selecting treatment plans and provide more individualized care to each patient.

Keywords: Dental caries, diagnosis, ICDAS, ICCMS

\section{INTRODUCCIÓN}

La caries dental es aún una de las enfermedades más prevalentes a nivel de salud pública, y las poblaciones de bajo nivel socioeconómico y cultural son las más vulnerables. En el mundo, más del $90 \%$ de los niños han sido afectados por esta enfermedad $\left({ }^{1}\right)$.

Citar como: Jara-Porroa JJ, De la CruzSedano GS, Ventura-Flores AK, PeronaMiguel de Priego GA. Herramientas actuales para el diagnóstico, manejo y control de la caries dental. parte II. Una revisión de la literatura. Rev Cient Odontol (Lima). 2020; 8(1): e007.

DOI: $10.21142 / 2523-2754-0801-2020-007$

La caries dental en niños tiene un patrón único y plantea desafíos completamente nuevos. Se trata de la enfermedad crónica infantil más común y es cinco veces más frecuente que el asma y siete veces más frecuente que la rinitis alérgica $(2,3)$. Los niños con caries de infancia temprana pueden tener bajo peso, sufrir deficiencia

\footnotetext{
División de Odontopediatría, Carrera de Estomatología, Universidad Científica del Sur. Lima, Perú.
} 
de hierro y crecer a un ritmo más lento debido a las molestias asociadas al comer por el dolor presentado $\left(^{2}\right)$.

El Sistema Internacional de Clasificación y Manejo de Caries (ICCMS) es un conjunto completo de protocolos clínicos que ayuda a tomar todas las decisiones de diagnóstico, prevención y tratamiento, con el fin de preservar la estructura del diente y restaurarlo solo cuando sea necesario $\left({ }^{4}\right)$.

El ICCMS tiene como objetivo entregar un método estandarizado para la clasificación y el manejo integral de la caries dental, a partir de otras herramientas como ICDAS, y de esta manera controlar el manejo de las lesiones de caries iniciales para evitar su progresión, así como restaurar de la manera más conservadora posible las lesiones de caries extensas para preservar, en lo posible, la mayor cantidad de la estructura dental. Pero este sistema reconoce que existen maneras diferentes de implementarlo localmente $(5,6)$.

Cabe destacar que no es el único sistema disponible en la actualidad que promueve el manejo del proceso de la caries y la evaluación de riesgos $\left(^{6}\right)$.

El objetivo de esta revisión es dar a conocer a la comunidad odontológica las nuevas herramientas para el control y manejo de caries dental, como el CAMBRA y el ICDAS, que ayudarán a su detección precoz y la estimación de su gravedad, además de permitir una atención individualizada para tratar y prevenir la enfermedad.

En esta segunda parte, se desarrollará el Sistema Internacional de Clasificación y Manejo de Caries (ICCMS).

\section{Sistema Internacional de Clasificación y Manejo de Caries (ICCMS)}

El ICCMS es un sistema enfocado que busca mantener la salud y cuidar la estructura dental. Evalúa la progresión de caries y la actividad de las lesiones, para así elaborar un plan de tratamiento preventivo ajustado al riesgo $\left({ }^{4,5}\right)$.
Este sistema se remonta al 2002, cuando varios grupos de investigadores se reunieron y crearon el Sistema Internacional de Detección y Valoración de Caries (ICDAS), el cual clasifica las lesiones de caries, con base en su aspecto visual clínico, para un diagnóstico adecuado, el pronóstico y el tratamiento clínico, tanto a nivel de salud individual como pública. El grupo ICDAS reconoció la caries dental como un desafío cambiante para clínicos y epidemiólogos/investigadores $\left({ }^{5,7}\right)$.

E1 ICCMS se construyó a partir del ICDAS, y pasó por una serie de talleres internacionales y simposios, para un entendimiento contemporáneo y un mejor manejo de la caries dental $\left({ }^{5}\right)$.

Para fines educativos, explicaremos el ICCMS mediante un diagrama en el cual se muestra que la secuencia de manejo de la caries es cíclica, ya que cada elemento es seguido por otro. El ciclo se reinicia después de cada cita (fig. 1).

Evaluación integral \& Plan personalizado de manejo de caries ICCMS ${ }^{\mathrm{TM}}$

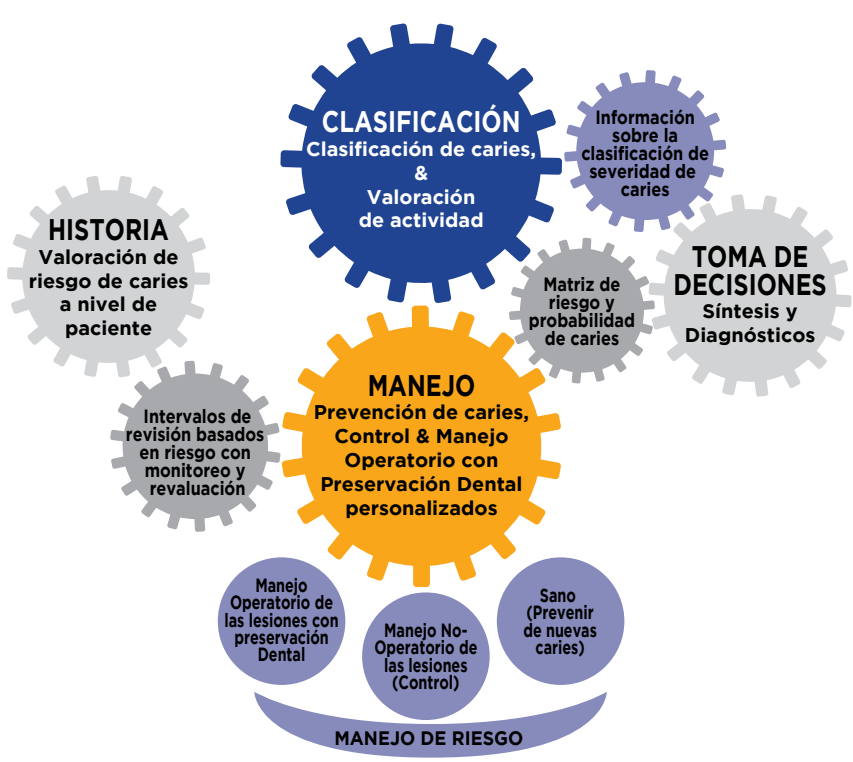

Figura I. Los cuatro elementos de ICCMS, diagramados con la descripción detallada de sus componentes y conectados por la cita de revisión basada en riesgo (tomado de Pitts N, Ismail A, Martignon S, Ekstrand K, Douglas G, Longbottom C). Guía ICCMS TM para clínicos y educadores. Sistema de Clasificación y Manejo de Caries (ICCMSTM). Junio de 2013 ( $\left.{ }^{5}\right)$. 


\section{ELEMENTOS DEL ICCMS}

Se divide en 4 elementos, que siguen el orden en que el clínico seguiría comúnmente la secuencia de manejo de caries $\left({ }^{5}\right)$.

\section{Elemento 1: historia. Valoración de riesgo de caries a nivel del paciente}

En este elemento valoraremos la historia clínica del paciente, se evalúa la cavidad oral y si existe de dolor. Una vez definido todo esto, evaluaremos los factores de riesgo, como uso de medicamentos, dieta, si tuvo terapia de radiación, higiene oral, condición socioeconómica e historia de caries de los padres $\left(^{5}\right)$.

Elemento 2: clasificación. Clasificación de caries y valoración de actividad de las lesiones con evaluación del riesgo intraoral de caries

Este elemento describe la evaluación clínica de la caries, que clasifica la severidad de la lesión y evalúa su actividad. Asimismo, se valoran los factores de riesgo intraoral de caries $\left({ }^{5,6}\right)$.

La valoración de caries siempre se efectuará mediante un examen visual (ICDAS) y debe combinarse con el examen radiográfico. Esto conduce a la obtención de información sobre la clasificación de la caries (inicial, moderada o severa) y el estado de actividad de la lesión (activa o inactiva) $\left.{ }^{5,6}\right)$.

\section{Clasificación de las lesiones}

El sistema ICCMS se basa en el ICDAS, que es un sistema de evaluación clínica que se utiliza para detectar la caries dental y evaluar las primeras etapas de la caries. Se emplea en la odontología actual, aplicaciones clínicas, investigaciones y estudios epidemiológicos. ICDAS se puede aplicar en superficies dentales y de raíz para detectar caries de esmalte, caries dentales, lesiones no cavitadas (manchas blancas) y lesiones cavitadas, y evaluar estas lesiones. Su objetivo es obtener información de mayor calidad para tomar mejores decisiones en el diagnóstico, pronóstico y manejo clínico de la caries dental, tanto a nivel individual como epidemiológico $\left.{ }^{8-10}\right)$.

E1 ICDAS presenta codificación del 0-6 y esta cifra depende de la gravedad de la lesión. El código 0 indica ausencia de evidencia de caries; el 1 y el 2, presencia de mancha blanca; el 3, fractura de esmalte; el 4, sombra oscura; el 5, socavado de esmalte de menos del $50 \%$ de la superficie, y el 6 , socavado en más del $50 \%$ de la superficie (tabla 1) $\left.{ }^{5,11}\right)$.

\section{Clasificación radiográfica de las lesiones coronales de caries}

E1 ICCMS clasifica radiográficamente las superficies posteriores de los dientes. Se ha reportado que tanto la reproducibilidad como la precisión de este sistema de clasificación son de significativas a excelentes. La
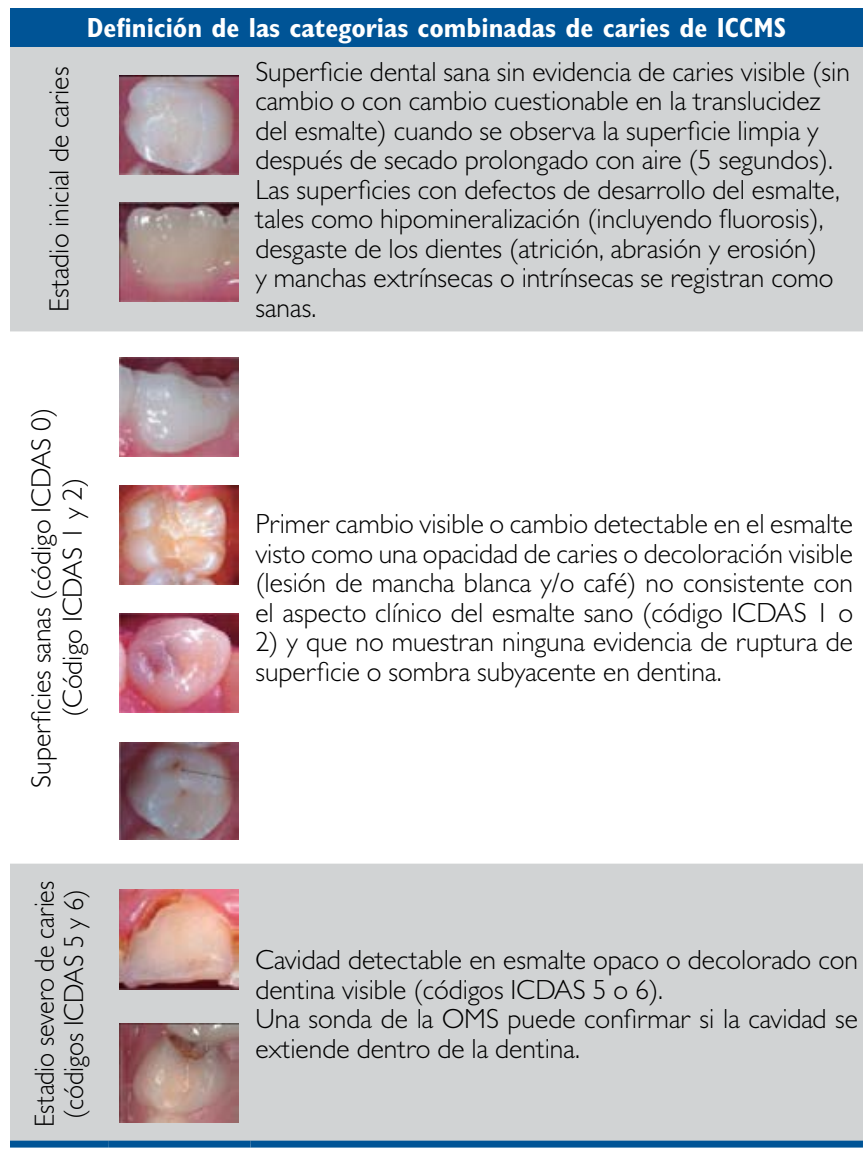

Tabla I. Tomado de Pitts N, Ismail A, Martignon S, Ekstrand K, Douglas G, Longbottom C. Guía ICCMS ${ }^{\top M}$ para clínicos y educadores. Sistema de Clasificación y Manejo de Caries (ICCMSTM), junio de $2013\left(^{5}\right)$. 
evidencia indica que la profundidad de penetración radiográfica en la que se puede predecir de forma confiable que la superficie del diente está cavitada y que la dentina está muy infectada es cuando la radiolucidez está más allá del tercio externo de dentina. Esto corresponde a los códigos 4, 5 y 6 en el sistema de clasificación radiográfica de ICCMS (tabla 2) $\left(^{5,6}\right)$.

\section{Combinación de la información clínica y radiográfica}

Se clasifica mediante las categorías inicial, moderada y severa (tabla 4).

Después de haber combinado la evaluación clínica con la radiográfica, evaluaremos la actividad de la lesión cariosa. Para esto, la dividiremos en lesión activa inicial o moderada y observaremos que la superficie del esmalte se presenta entre blancuzca o amarillenta, opaca, con pérdida de brillo y rugosa. La lesión aparece un área

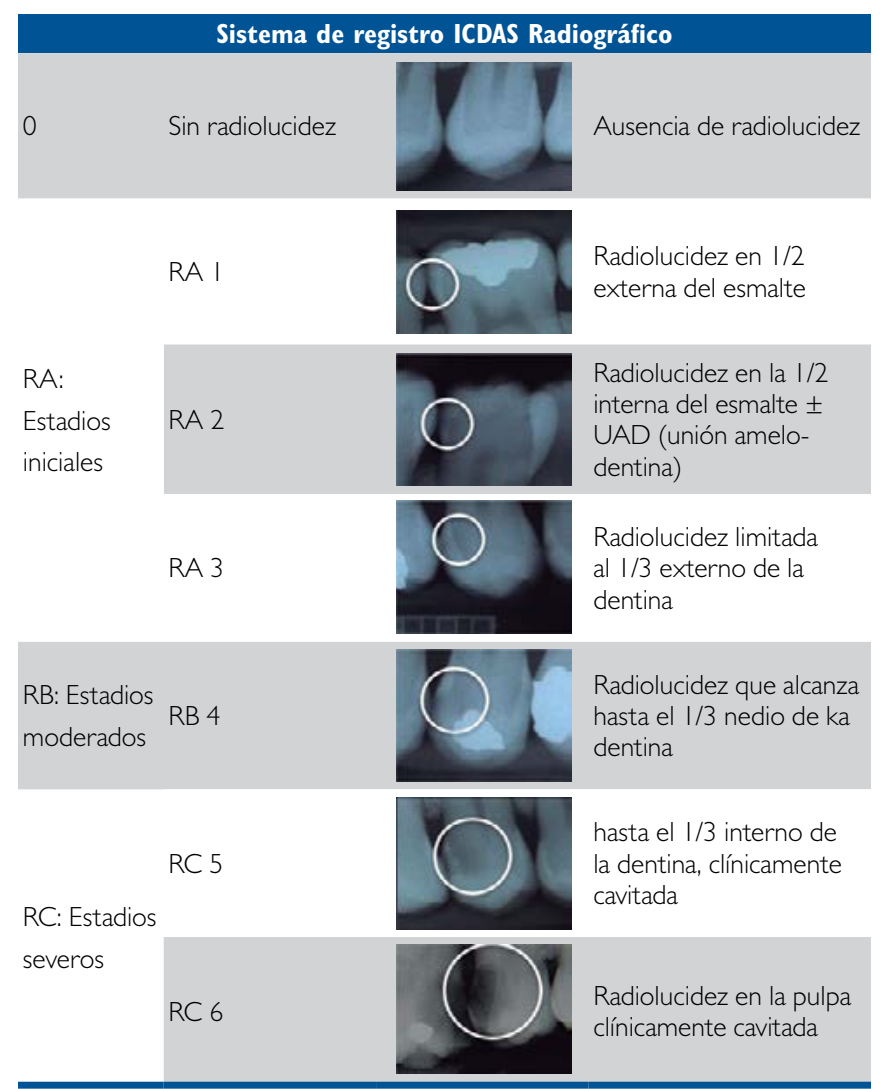

Tabla 2. Tomado de Pitts N, Ismail A, Martignon S, Ekstrand K, Douglas G, Longbottom C. Guía ICCMS TM para clínicos y educadores. Sistema de Clasificación y Manejo de Caries (ICCMSTM), junio de $2013\left(^{5}\right)$. retentiva de placa dental. En estadios severos, la lesión activa será suave al sondaje y sentiremos la dentina blanda o con una consistencia tipo cuero $\left.{ }^{5,7}\right)$.

En el caso de la lesión inactiva en estadio inicial o moderado, se observa que la superficie del esmalte es blancuzca, amarillenta o negra; el esmalte puede estar brillante, duro y liso. Para superficies interproximales, la lesión de caries típicamente se localiza a cierta distancia del margen gingival. La lesión puede no estar inicialmente cubierta por placa gruesa. En los estadios severos de lesiones inactivas, la dentina será brillante $\mathrm{y}$ dura al sondaje suave $\left(^{5,7}\right)$.

\section{Elemento 3: Toma de decisiones. Síntesis de la información para establecer el diagnóstico}

E1 ICCMS presenta dos formas de decisión clave que ayudan a los dentistas a desarrollar planes de tratamiento

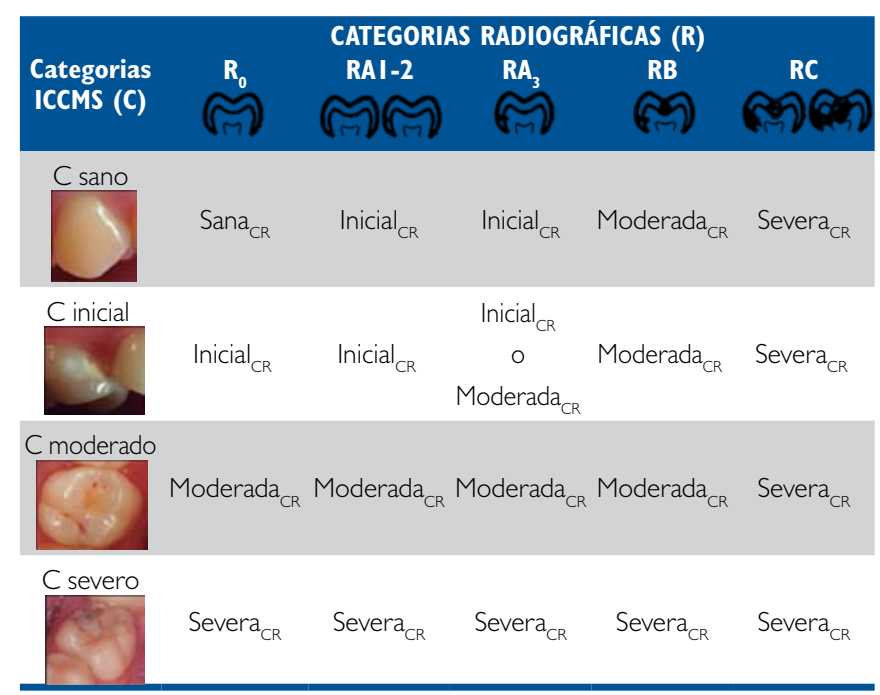

Tabla 3. Tomado de Pitts N, Ismail A, Martignon S, Ekstrand K, Douglas G, Longbottom C. Guía ICCMS TM para clínicos y educadores. Sistema de Clasificación y Manejo de Caries (ICCMSTM), junio de $2013\left({ }^{5}\right)$.

\begin{tabular}{ccc|} 
Codigos de caries & \multicolumn{2}{c|}{ Estado de actividad } \\
& Lesiones activas & Lecciones inactivas \\
\hline ICCMSTM inicial & Inicial activa & Inicial inactiva \\
\hline ICCMSTM moderada & Moderada activa & Moderada inactiva severa \\
\hline ICCMSTM severa & Activa severa & Inactiva \\
\hline
\end{tabular}

Tabla 4. Tomado de Pitts N, Ismail A, Martignon S, Ekstrand K, Douglas G, Longbottom C. Guía ICCMS TM para clínicos y educadores. Sistema de Clasificación y Manejo de Caries (ICCMSTM), junio de 2013. 


\begin{tabular}{ccccc} 
Matriz ICCMS de riesgo de caries y probabilidad & $\begin{array}{c}\text { Ausencia de lesiones } \\
\text { de caries activas* }\end{array}$ & $\begin{array}{c}\text { Lesiones de caries en } \\
\text { estado inicial activas }\end{array}$ & $\begin{array}{c}\text { Lesiones de caries en estado } \\
\text { moderado o severo activas Estado }\end{array}$ \\
\hline Riesgo bajo & Probabilidad baja & Probabilidad moderada & Probabilidad moderada* \\
Riesgo moderado & Probabilidad baja & Probabilidad moderada & Probabilidad alta \\
Riesgo alto & Probabilidad moderada & Probabilidad alta & Probabilidad alta \\
\hline
\end{tabular}

Tabla 5. Matriz ICCMS de riesgo de caries y probabilidades. Tomado de Pitts N, Ismail A, Martignon S, Ekstrand K, Douglas G, Longbottom C. Guía ICCMS TM para clínicos y educadores. Sistema de Clasificación y Manejo de Caries (ICCMSTM), junio de $2013\left(^{5}\right)$.

\begin{tabular}{|c|c|c|c|}
\hline \multicolumn{2}{|l|}{ Superficie } & \multirow[b]{2}{*}{$\begin{array}{l}\text { Mesial-distal } \\
\text { (proximal) }\end{array}$} & \multirow[b]{2}{*}{ Superficies lisas } \\
\hline $\begin{array}{l}\text { Estadío } \\
\text { ICCMS }^{\mathrm{TM}}\end{array}$ & Fosas y fisuras & & \\
\hline M Sano & \multicolumn{3}{|c|}{ Prevención basada en riesgo } \\
\hline & \multicolumn{3}{|c|}{$\begin{array}{l}\text { MNO: Fluoruro tópico aplicado clínicamente; barniz de fluoruro } \\
\text { recomendado para niños } \leq 6 \text { años. }\end{array}$} \\
\hline M Inicial Activa & $\begin{array}{l}\text { MNO: Sellantes } \\
\text { a base de resina / } \\
\text { ionómero de vidrio. }\end{array}$ & $\begin{array}{l}\text { MNO: Sellantes a base } \\
\text { de resina / infiltrantes. }\end{array}$ & \\
\hline
\end{tabular}

MNO: Higiene oral con crema dental fluorada ( $\geq 1000$ ppm) cuando erupciona el primer diente. Se recomienda supervisión por lo menos hasta la edad de 8 años.

\begin{tabular}{|c|c|c|c|}
\hline $\begin{array}{l}\text { M Inicial } \\
\text { Detenida }\end{array}$ & \multicolumn{3}{|c|}{ Sin tratamiento específico para la lesión } \\
\hline \multirow{3}{*}{$\begin{array}{l}\text { M Moderada } \\
\text { Activa }\end{array}$} & $\begin{array}{l}\text { MNO: Sellantes a } \\
\text { base de resina* }\end{array}$ & & $\begin{array}{l}\text { MNO: Sellantes a } \\
\text { base de resina* }\end{array}$ \\
\hline & $\begin{array}{l}\text { MNO: Si no es } \\
\text { factible el sellante } \\
\text { (dificultad para aislar } \\
\text { el diente) una opción } \\
\text { es la corona metálica } \\
\text { o forma plástica } \\
\text { preformadas sin } \\
\text { preparación dental. }\end{array}$ & & $\begin{array}{l}\text { MNO: Si es sellante } \\
\text { no es viable (dificultad } \\
\text { para aislar el diente) } \\
\text { una opción es la } \\
\text { corona metálica } \\
\text { o forma plástica } \\
\text { preformadas sin } \\
\text { preparación dental. }\end{array}$ \\
\hline & $\begin{array}{l}\text { MOPD: Incluyendo } \\
\text { la colocación de } \\
\text { corona metálica } \\
\text { o forma plástica } \\
\text { preformadas }\end{array}$ & $\begin{array}{l}\text { Para opciones de } \\
\text { manejo apropiadas } \\
\text { determinar la } \\
\text { presencia de cavidad } \\
\text { por separación dental. } \\
\text { Si no hay cavidad: } \\
\text { MNO. Si hay cavidad: } \\
\text { MOPD (incluyendo } \\
\text { corona metálica } \\
\text { o forma plástica } \\
\text { preformadas) }\end{array}$ & $\begin{array}{l}\text { MOPD: incluyendo } \\
\text { la colocación de } \\
\text { corona metálica } \\
\text { o forma plástica } \\
\text { preformadas. }\end{array}$ \\
\hline $\begin{array}{l}\text { M Moderada } \\
\text { Detenida }\end{array}$ & \multicolumn{3}{|c|}{$\begin{array}{l}\text { MOPD si la lesión es un ARP o el área es estéticamente } \\
\text { inaceptable. }\end{array}$} \\
\hline \multirow{2}{*}{$\begin{array}{l}\text { M Severa } \\
\text { Activa }\end{array}$} & \multicolumn{3}{|c|}{ MOPD (incluyendo corona metálica o forma plásticas preformadas) } \\
\hline & \multicolumn{3}{|c|}{$\begin{array}{c}\text { Si el manejo restaurativo no es posible, considerar Técnica de Hall } \\
\text { o extracción. }\end{array}$} \\
\hline $\begin{array}{l}\text { M Severa } \\
\text { Detenida }\end{array}$ & \multicolumn{3}{|c|}{$\begin{array}{c}\text { MOPD si la lesión es un ARP o el área es estéticamente } \\
\text { inaceptable. }\end{array}$} \\
\hline
\end{tabular}

MNO = Manejo no operatorio. MOPD = Manejo operatorio con preservación dental. ARP $=$ Área retentiva de placa* Si el manejo restaurador preferido no es factible debido a factores del paciente o del diente, el tratamiento alternativo es la aplicación de un sellante a base de ionómero de vidrio.

Tabla 6. Tomada de Pitts N, Ismail A, Martignon S, Ekstrand K, Douglas G, Longbottom C. Guía ICCMS TM para clínicos y educadores. Sistema de Clasificación y Manejo de Caries (ICCMSTM), junio de $2013\left({ }^{5}\right)$. para administrar el estado de riesgo de caries y proporcionar atención preventiva y restaurativa. Se utiliza la información basada en las lesiones de caries y los factores de riesgo que tiene el paciente para continuar con la enfermedad. En este elemento, haremos la síntesis y el diagnóstico para el futuro plan de tratamiento $\left({ }^{5,6}\right)$.

El análisis de riesgo de caries de ICCMS valora la probabilidaddedesarrollodenuevaslesionesolaprogresión de la caries (tabla 5). Esto implica que a los individuos se les clasifique como de riesgo bajo, moderado o alto $\left(^{5,6}\right)$.

\section{Elemento 4: Manejo. Prevención de caries, control y manejo operatorio con preservación dental personalizados}

Después de haber evaluado la actividad y el riesgo a desarrollar caries dental. Se elaborará un plan de tratamiento integral e individualizado para cada paciente (figura 2) ( ${ }^{5}$ ).

Para el manejo de las lesiones ya existentes, utilizaremos desde tratamientos preventivos hasta invasivos para inactivar las lesiones. Para controlar la probabilidad de desarrollar nuevas lesiones, trabajaremos con los factores de riesgo revisando desde la primera cita el manejo de la dieta y la higiene dental (tabla 6).

\section{DISCUSIÓN}

E1 protocolo ICCMS es actualmente el sistema más completo para el manejo y prevención de la caries, ya que permite tomar las decisiones correctas para preservar la estructura dentaria y restaurar solo cuando sea necesario; sin embargo, es importante saber que no es el único sistema disponible $\left(^{4}\right)$. 


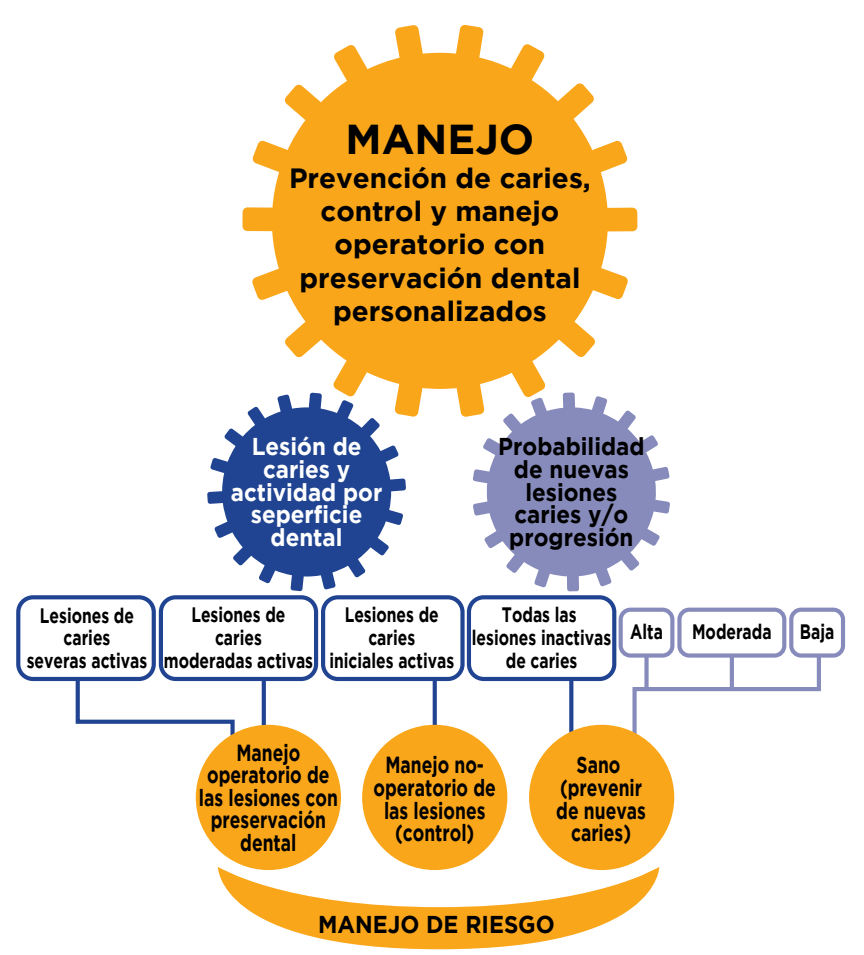

Figura 2. Manejo de caries dental. Tomado de Pitts N, Ismail A, Martignon $\mathrm{S}$, Ekstrand K, Douglas G, Longbottom C. Guía ICCMS TM para clínicos y educadores. Sistema de Clasificación y Manejo de Caries (ICCMSTM), junio de $2013\left({ }^{5}\right)$.

No hay estudios que evalúen el sistema ICCMS a largo plazo, pero se están realizando estudios comparativos para evaluar el proceso ylos resultados de su implementación $\left({ }^{4}\right)$.

Ismail et al. indican que la ruta para el manejo de la caries según ICCMS es un protocolo que ayuda a los profesionales a recopilar y analizar los datos personales y clínicos del paciente para desarrollar planes integrales de atención que permitirán cambiar el comportamiento de los pacientes, de modo que disminuyan los riesgos y manejen acciones preventivas en su hogar. La ruta del manejo de caries consiste en prevenir, controlar y restaurar la caries dental. ICCMS integra en sus procedimientos la filosofía de CAMBRA; sin embargo, a diferencia de ella, presenta una descripción detallada y más completa para el manejo de la caries $\left({ }^{4}\right)$.
Pitts y Ekstrand (2013) proporcionan una visión general del Sistema Internacional de Detección y Evaluación de Caries (ICDAS) y su Sistema Internacional de Clasificación y Gestión de Caries (ICCMS). Ellos describen y hacen referencia a los pasos clave en el desarrollo de estos sistemas, explican la evolución de estos sistemas durante la última década y explican cómo se están utilizando para para que los dentistas puedan manejar la caries de manera adecuada $\left({ }^{7}\right)$.

ICDAS emplea un enfoque basado en evidencia y está orientado a la prevención. Es un sistema de detección y evaluación que clasifica las etapas del proceso de caries en función de su extensión y actividad histológica; está diseñado para su uso en los cuatro dominios de práctica clínica, educación, investigación y salud pública. Luego de más de una década, ICDAS ha evolucionado y ha generado el ICCMS para permitir mejores resultados en el tema de caries a largo plazo. ICDAS proporciona métodos flexibles y cada vez más adaptados internacionalmente para clasificar las etapas del proceso de caries y el estado de actividad de las lesiones que puede incorporarse al ICCMS. El ICCMS proporciona opciones para integrar y sintetizar información de pacientes y sus dientes, incluyendo el estado de riesgo de caries, con el fin de planificar, gestionar y revisar la caries en clínica y práctica de salud pública.

Contribución de autoría: Jackeline Jajaira Jara-Porroa, Gabriela Silvia De la Cruz-Sedano y Abigail Katherine Ventura-Flores han participado en la concepción del artículo, la recolección de información, su redacción y aprobación de la versión final. Guido Perona Migueldel Priego participó en la concepción del artículo y la aprobación de la versión final.

Fuente de financiamiento: Autofinanciado.

Potenciales conflictos de interés: Los autores declaran no tener conflicto de interés de ningún tipo. 


\section{REFERENCIAS BIBLIOGRÁFICAS}

1. Arangannal P, Mahadev SK, Jayaprakash J. Prevalence of dental caries among school children in Chennai, Based on ICDAS II. J Clin Diagn Res. 2016; 10 (4): ZC09-ZC12.

2. Koya S, Ravichandra KS, Arunkumar V, Sahana S, Pushpalatha HM. Prevalence of early childhood caries in children of West Godavari District, Andhra Pradesh, South India: An epidemiological study. Int J Clin Pediatr Dent. 2016; 9 (3): 2515. doi: 10.5005/jp-journals-10005-1372

3. Gugnani N, Pandit IK, Srivastava N, Gupta M, Sharma M. International Caries Detection and Assessment System (ICDAS): A New Concept. Int J Clin Pediatr Dent. 2011; 4 (2): 93-100. doi: 10.5005/jp-journals-10005-1089

4. Ismail A, Téllez M, Pitts NB, et al. Caries management pathways preserve dental tissues and promote oral health. Community Dent Oral Epidemiol. 2013, 41 (1): e12-e40.

5. Pitts N, Ismail A, Martignon S, Ekstrand K, Douglas G, Longbottom C. Guía ICCMS ${ }^{\mathrm{TM}}$ para clínicos y educadores. Londres: ICDAS Foundation; 2013.

6. Ismail A, Pitts NB, Téllez M. The International Caries Classification and Management System $\left(\mathrm{ICCMS}^{\mathrm{TM}}\right)$ an example of a Caries Management Pathway. BMC Oral Health 2015; 15 (Suppl 1): S9. doi: 10.1186/1472-6831-15-S1-S9
7. Pitts NB, Ekstrand KR. International Caries Detection and Assessment System (ICDAS) and its International Caries Classification and Management System (ICCMS) - methods for staging of the caries process and enabling dentists to manage caries. Community Dent Oral Epidemiol. 2013; 41: e41-e52.

8. Dikmen B. ICDAS II criteria (international caries detection and assessment system). J Istanbul Univ Fac Dent 2015; 49 (3): 63 72.

9. Ismail AI, Sohn W, Tellez M, et al. The International Caries Detection and Assessment System (ICDAS): an integrated system for measuring dental caries. Community Dent Oral Epidemiol. 2007;35(3):170-178. doi: 10.1111/j.16000528.2007.00347.x

10. Neuhaus KW, Jost F, Perrin P, Lussi. Impact of different magnification levels on visual caries detection with ICDAS. J Dent. 2015; 43 (12): 1559-64. doi: 10.1016/j.jdent.2015.09.002

11. Fernandes I, Sá-Pinto A, Silva L, Ramos-Jorge J, Ramos-Jorge M. Maternal identification of dental caries lesions in their children aged 1-3 years. Eur Arch Paediatr Dent 2017; 18 (3): 197-202. doi: 10.1007/s40368-017-0286-8 